Please do not remove this page

RMIT

UNIVERSITY

\title{
A Space-Time MIMO Channel Model for Macrocell Mobile Environment
}

Al-Qahtani, Fawaz; Mahmoud, Seedahmed; Hussain, Zahir

https://researchrepository.rmit.edu.au/esploro/outputs/9921862523801341/filesAndLinks?institution=61RMIT_INST\&index=null

Al-Qahtani, F., Mahmoud, S., \& Hussain, Z. (2006). A Space-Time MIMO Channel Model for Macrocell Mobile Environment. 12th Asia Pacific Conference on Communications.

https://doi.org/10.1109/APCC.2006.255852

Published Version: https://doi.org/10.1109/APCC.2006.255852

Repository homepage: https://researchrepository.rmit.edu.au

(c) 2006 IEEE. Personal use of this material is permitted. However, permission to reprint/republish this material for advertising or promotional purposes or for creating new collective works for resale or redistribution to servers or lists, or to reuse any copyrighted component of this work in other works must be obtained from the IEEE.

Downloaded On 2023/04/27 00:46:55 +1000 


\title{
A Space-Time MIMO Channel Model for Macrocell Mobile Environment
}

\author{
Fawaz S. Al-Qahtani, Seedahmed S. Mahmoud, Zahir M. Hussain, SMIEEE \\ School of Electrical and Computer Engineering \\ RMIT University, Melbourne, Victoria 3000, Australia. \\ E-mails:s3115478@student.rmit.edu.au,mahmoud.seedahmed@rmit.edu.au,zmhussain@ieee.org
}

\begin{abstract}
This paper introduces a general concept of a space time geometrical channel model with hyperbolically distributed scatterers for a macrocell mobile environment. This model provides statistics of the time of arrival (TOA) and direction of arrival (DOA). The proposed channel model is modified to Multiple Input Multiple Output (MIMO) model which characterizes the space and time-variance characters of the mobile radio channel. Our MIMO channel model is based on one-ring scattering model. This model is examined under different mobile environments. Moreover, we studied the influence of the physical parameters (such as scatterers distribution) as well as the system parameters (such as antennas spacing) on the capacity.
\end{abstract}

\section{INTRODUCTION}

Nowadays, the demand for high data rates has increased for transmission systems. For that, the attention has been paid to multiple-input multiple-output (MIMO) broadband wireless system. The MIMO channel can be seen as parallel spatial subchannels that allow the transmission of parallel data streams. Deploying multiple antennas at both transmitter and receiver increases the capacity linearly with the number of spatial subchannels via potential decorrelation between the subchannels [1],[2]. The MIMO channel can provide diversity gain, array gain, and interference canceling gain. These gain can be achieved using spatial multiplexing alogrithms (example "BLAST") and Space Time block coding (STBC) [10], [11], [16], [17].

On the other hand, the channel modelling is playing very important role in mobile communication system design. It can be divided into wideband and narrowband models. The wideband model treats the propagation channel as frequencyselective, which means that different frequency sub-bands have different channel responses, while the narrowband models assume that channel has a frequency non-selective fading [4].

There are many parameters describe channel model such as received signal strength, Doppler spectra and power delay profiles (PDP). A statistical channel parameters is required such as directional of arrival (DOA) and time of arrival (TOA) of the multipath components. On the other hand, scalar stochastic channel models which provide time variant fading are not directly applicable for systems with multiple antennas [5],[6]. For this reason, spatial channel model is required to characterize the space and time-variant effects of the mobile radio channel. Surprisingly, the simulation of MIMO propagation system can assist in the choice of efficient modulation scheme under different scenarios.

Generally, the MIMO channel modelling can be divided into three different categories: scattering, ray-tracing, and correla- tion models. In this paper, the MIMO channel model is based on the first type (scatering) model. Currently there are many models have presented based on geometrical distributions of scatterers and such models are based on the assumptions that the channel wave between transmit and receive antennas is propagating through a single bounce scattering and there is no refraction and diffraction such models are known as Geometrical Based Single Bounce (GBSB) models, the ring model [8], uniform model [14], circular model [15] and elliptical model [12]. In [15], the scatterer is assumed constant within a circular area around the mobile station (MS) and in [12] the scatter density is assumed to be constant with an elliptical region about the mobile and the base station (BS).

Organization of the Paper: In Section II, we introduce the general model and review its mathematical background. In Section III, we present the MIMO geometrical model. Section IV provides a performance of the capacity including the influence of the physical parameters. Section. $V$ contains numerical results and Section VI concludes the paper.

\section{General Geometrical Model}

Each channel has different multipaths which can be modelled by its dominant path that represent the maximum energy of a path cluster. In this section, $M_{T}$ transmit and $M_{R}$ receive antennas will be deployed. For each multipath component, AOA and AOD are defined with respect to the model which is intended to be used. A geometrical-based Macrocell channel (GBMC) [6] is used which is based on the following assumptions:

- There is no LOS between mobile and base station for the macro-cell environments.

- Signal received at the based station (BS) is propagating along the horizon and no vertical components are involved

- The channel wave is propagating through a single bounce scattering and there is no refraction and diffraction waves occurring.

- Scatterers' reacts as an omni-directional re-radiation elements, whereby the plane wave is reflected to the base station without any influence from other scatterers.

- It is assumed that there is no signal scattering around the base station. The antenna heights which are relatively high and there is no signal scattering from locations near the base station where $R<D$

- This model assumes that the scatterers are arranged circularly around the mobile with the distances between 
the mobile and scatters are distributed hyperbolically according to inverse-cosh-squared distribution.

The probability density function of the distance $r_{k}$ has the form [6],[7].

$$
f_{r_{k}}\left(r_{k}\right)= \begin{cases}\frac{1}{B \cosh ^{2}\left(a r_{k}\right)} & \text { for } 0 \leq r_{k} \leq R \\ 0 & \text { elsewhere }\end{cases}
$$

where $R$ is the radius of the scatterers' circle, $a$ is the spread control parameter lies in the interval $(0,1)$ which controls the spread (standard deviation) of the scatterers around the mobile. $B$ is the normalization factor for the pdf which is given by $\tanh (a R) / a$. This leads to the final pdf form of the hyperbolical distribution of the scatterers which is given by

$$
f_{r_{k}}\left(r_{k}\right)= \begin{cases}\frac{a}{\tanh (a R) \cosh ^{2}\left(a r_{k}\right)} \quad \text { for } 0 \leq r_{k} \leq R \\ 0 \quad \text { elsewhere }\end{cases}
$$

This model provides the statistics of DOA, TOA, phase data and amplitude of the multipath components. Many scatterers are assumed to be located around the mobile station,MB. A transmit signal from the mobile travels through number of paths where the number of paths can become very large if all possible paths are taken into account but from the practical point of view the paths that have high noise level will be neglected. The received signal multipaths arrive at the receiver with different DOAs. In this Section DOA and TOA will be derived based on GHSM model.

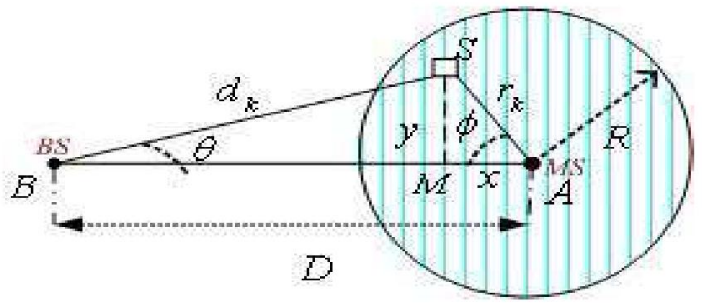

Fig. 1. General structure of Geometry of the Geometrical Hyperbolic Scattere Model

Fig. 1 shows a simple model representing one transmitter and one receiver. $D$ is the distance between the mobile and the base station, $S$ is the scatterer, both $x$ and $y$ represent the location of scatterer, $\phi$ is the angle of departure which is assumed to be uniformly distributed over $(0,2 \pi)$. $R$ is the radius of the ring $D<R$, and $\theta$ is direction of arrival. The probability density of the angle of departure $\phi$ is

$$
f_{\phi}\left(\phi_{k}\right)=\frac{1}{2 \pi} \quad \text { for } 0 \leq \phi \leq 2 \pi
$$

Using Cumulative Distribution Function (CDF), both $r_{k}$ and $\phi_{k}$ can be obtained from random variables $x$ and $y$.

$$
\begin{gathered}
r_{k}=\frac{1}{a} \arctan [x \tanh (a R)] \\
\phi_{k}=2 \pi y
\end{gathered}
$$

The location of scatterer $x_{k}$ and $y_{k}$ can be obtained with respect to the angle of departure $\phi_{k}$ and the direction of scatterer $r_{k}$. The distance between the scatterer and the base station is given by

$$
d_{k}=\sqrt{r_{k}^{2}+D^{2}-2 r_{k} \cos \left(\phi_{k}\right)}
$$

The total distance between the mobile and the base station is summation of the two paths from mobile to scatter $r_{k}$ and from the scatterer $r_{k}$ to the base station $d_{k}$, these two paths can be described by free space propagation models since no interfering factors are considered in "single-bounce" models. After that, total delay path $\tau_{k}$ of the $k^{t h}$ multipath can be derived immediately from 3,4 and 5 ; which has the following form:

$$
\tau=\frac{d_{k}+r_{k}}{c}=\frac{1}{c}\left[r_{k}+\sqrt{r_{k}^{2}+D^{2}-2 r_{k}-2 r_{k} \cos \left(\phi_{k}\right)}\right]
$$

This delay describes the time that has been taken to reach the receivers via a single bounce. The direction of arrival $\theta_{k}$ of the $k^{\text {th }}$ multipath components is derived using trigonometric functions which applied to the model in Fig.1, the DOA is given by

$\theta_{k}= \begin{cases}\arctan \left(\frac{r_{k} \sin \left(\phi_{k}\right)}{D-r_{k} \cos \left(\phi_{k}\right)}\right) & \text { for } r_{k} \cos \left(\phi_{k}\right) \leq D \\ \arctan \left(\frac{r_{k} \sin \left(\phi_{k}\right)}{D-r_{k} \cos \left(\phi_{k}\right)}\right)+\pi & \text { for } r_{k} \cos \left(\phi_{k}\right)>D\end{cases}$

The mean power of each multipath component depends on the multipaths delay $\tau_{k}$ which is defined by a characteristic power delay profile (PDP); it is given by

$$
P\left(\tau_{k}\right)=P_{\text {ref }}-10 n \log \left(\frac{\tau_{k}}{\tau_{\text {ref }}}\right)
$$

where $n$ is the path loss exponent and $P_{r e f}$ is reference power that is measured at a distance $d_{r e f}$ from the transmitting antenna, $P_{r e f}$ is given by

$$
P_{\text {ref }}=P_{T}-20 \log \left(\frac{4 \pi d_{r e f} f_{c}}{c}\right)
$$

where $P_{T}$ is the transmitted power in $\mathrm{dB}$

\section{Geometrical MiMO Model}

We consider a single user, point to point communication with $n_{T}$ transmitting antenna and $n_{R}$ receiving antennas.The complex baseband system equation can generally be written as

$$
\mathbf{y}=\mathbf{H x}+\mathbf{z}
$$

where $\mathbf{x}$ is the transmit signal, $\mathbf{y}$ is the receive vector, and $\mathbf{z}$ is the noise vector. $\mathbf{H} \in \mathbf{C}^{\dot{R} \times \mathbf{T}}$ is the channel matrix that contains coefficient between $n_{T}$ transmit and $n_{R}$ receive antenna and all its entries are independently identically distributed (i.i.d) complex Gaussian random variables. For simplicity, uniform linear arrays (ULAs) are used as antenna geometry. The channel matrix can be described via steering 


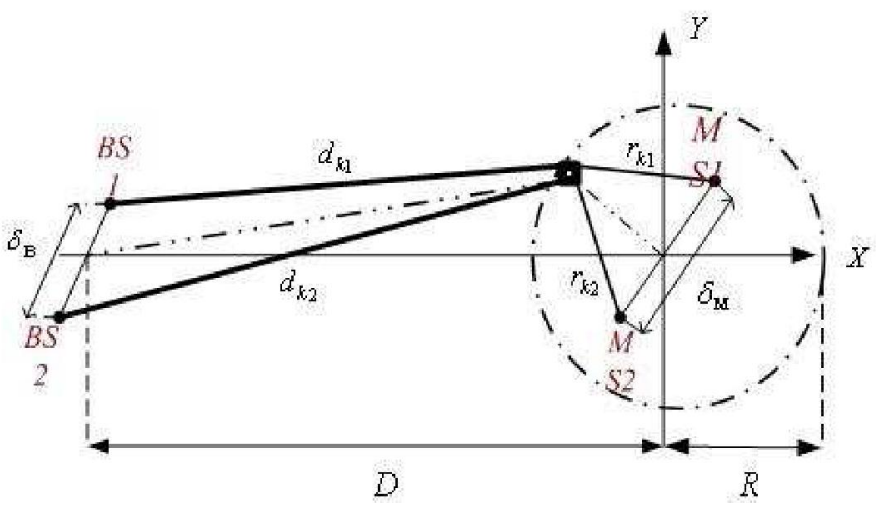

Fig. 2. One Ring MIMO GHSM Channel Model

vector denoting where $\delta_{T}$ and $\delta_{R}$ are the antenna spacing at the transmitter and receiver, respectively. They are given by:

$$
\begin{aligned}
& \mathbf{a}\left(\phi_{T}\right)=\frac{1}{\sqrt{N}}\left[1, e^{\frac{-j 2 \pi \delta_{T}}{\lambda} \sin \left(\phi_{T}\right)}, \cdots, e^{\frac{-j 2 \pi(N-1) \delta_{T}}{\lambda} \sin \left(\phi_{T}\right)}\right] \\
& \mathbf{a}\left(\theta_{R}\right)=\frac{1}{\sqrt{N}}\left[1, e^{\frac{-j 2 \pi \delta_{R}}{\lambda} \sin \left(\theta_{R}\right)}, \cdots, e^{\frac{-j 2 \pi(M-1) \delta_{T}}{\lambda} \sin \left(\theta_{T}\right)}\right]
\end{aligned}
$$

where $\mathbf{a}\left(\phi_{T}\right)$ represents the steering vector for the transmitter array to send a beam in the direction of $\phi_{T}$ and $\mathbf{a}\left(\theta_{R}\right)$ is the receive array response due to appoint source in the direction of $\theta_{R}$. For each significant multiparty components (MPC) the angle of arrival AOA and the angle of departure $\mathrm{AOD}$ are defined with respect to the array and the position of a dominate reflectors AOD is modelled as a uniform random distribution yielding the well-known Jakes Spectrum [8]. This model referees to non-physical model which based on the channel statistical characteristics. We consider MIMO channel as virtual channel model [8]. We assume there are $L$ scatterers within one cluster between the transmitter and the receiver and one physical MIMO channel for this cluster is

$$
h_{l, n, m}(t)=\frac{1}{\sqrt{L}} \sum_{l=0}^{L-1} \sqrt{P\left(\tau_{l}\right)} a_{m}\left(\theta_{l}^{R}\right) a_{n}\left(\phi_{l}^{T}\right) \beta_{l, m, n}(t)
$$

where $P$ is the average power of the $L^{t h}$ scatter and $\tau_{l}$ is the delay for the $L^{t h}$ scatters. The two array propagation phase shifts $a_{m}\left(\theta_{l}{ }^{R}\right)$ and $a_{n}\left(\phi_{l}{ }^{T}\right)$ refer to the spatial characteristic of the fading process and $\beta_{l, m, n}(t)$ is the temporal correlation characteristic of the fast fading process.

$$
\beta_{l, m, n}=\alpha(t) e^{j 2 \pi f_{d} \cos \left(\phi_{l}^{T}-\gamma\right) t}
$$

where $\alpha(t)$ is the attenuation of scatter which is assumed to be resulted from a random process with variance $\sigma=1$ and $f_{d}$ is the maximum frequency shift (Doppler) which is equal to $f_{c} v / c, v$ being the velocity of the object ( $\gamma$ is the direction of the object) and $c$ is the speed of light. The correlation between signals form different antennas is referred to spatial fading correlation which can be defined at the transmitter Tx as:

$$
R_{n}^{T x}=\sum_{l=0}^{L-1} a_{n}\left(\phi_{l}^{T}\right) a_{n}^{H}\left(\phi_{l}^{T}\right)
$$

It is based on the antennas separation and AOD. High correlation can be observed for small antenna separation and low angular spreads. The correlated MIMO channel is a result of multiplying the $\mathrm{Tx}$ and $\mathrm{Rx}$ correlation by the channel [2],[3].

$$
\mathbf{H}=\mathbf{R}_{n}^{1 / 2} \mathbf{H}_{w} \mathbf{R}_{m}{ }^{1 / 2}
$$

where $\mathbf{H}_{w}$ is a $n_{T} \times r_{R}$ matrix of the complex Gaussian fading coefficients. The spatial covariance matrix is computed from different antenna array configurations and ULA is considered in this work.

\section{MIMO CAPACITY}

The channel capacity is considered under limitation of the system bandwidth and total transmit power $P_{T}$. It is a random quantity which is a function of the channel matrix and its distribution based on channel distribution [1],[2]. In this section, two expressions are defined and both are known at the receiver. First, transmitter has no knowledge about the channel (unknown-Tx) and transmitter has a full or partial knowledge about the transmitter and (known-Tx)

Unknown-Tx: the transmitted signal $x$ is composed of statistically independent equal power components each with a circularly symmetric complex Gaussian distribution. The power is distributed equally among the elements $N$ of the array at the transmit antennas [2].

$$
P_{n}=\frac{P_{T_{x}}}{N} \quad \text { for } n=1,2, \ldots, N
$$

The capacity is defined under power constraints

$$
\begin{aligned}
C & =\log _{2}\left(I_{r}+\frac{\rho}{n_{T}} H H^{H}\right) \\
& =\sum_{i=1}^{M} \log _{2} \operatorname{det}\left(1+\frac{\rho}{n_{T}} \lambda_{H, i}^{2}\right) \text { bits } / \mathrm{s} / \mathrm{Hz}
\end{aligned}
$$

where, $\rho$ is the signal to noise ratio SNR at each receiver antenna, $(.)^{H}$ denotes the complex conjugate transpose transform, det(.) is the determinant, $\mathbf{I}_{r}$ is $r \times r$ identity matrix and $M$ referes to $\min \left(n_{T}, n_{R}\right)$. It has been approved that spatial fading correlation degrades the capacity of the system [2]. From equations 17 and 19 , capacity is extended to

$$
C=\log _{2}\left(I_{r}+\frac{\rho}{n_{T}} R_{m}{ }^{1 / 2} H_{w} R_{n} H_{n}^{H} R_{m}{ }^{H}\right) \quad \text { bits } / \mathrm{s}
$$

Known-Tx: as the channel known at the transmitter more capacity can be achieved by distributing the power of the signal optimally over $n_{T}$ antennas. The power gain available at each $k^{t h}$ subchannel is given by the $k_{t h}$ eigenvalue. The SNR for the $k^{t h}$ subchannel is given by

$$
\rho_{k}=\lambda_{k} \frac{P_{k}}{\sigma_{n}^{2}}
$$


TABLE I

PATH LOSS EXPONENT FOR DIFFERENT ENVIRONMENTS

\begin{tabular}{|l|r|c|}
\hline Enviroment & Path loss exponent $\mathbf{n}$ & Simulation Parameters n \\
\hline \hline Free Space & 2 & 2 \\
\hline Urban area & $\mathbf{2 . 7}$ to 3.5 & 2.7 \\
\hline Shadowed urban & 3 to 5 & 4 \\
\hline Obstructed in factory & 4 to 6 & 6 \\
\hline
\end{tabular}

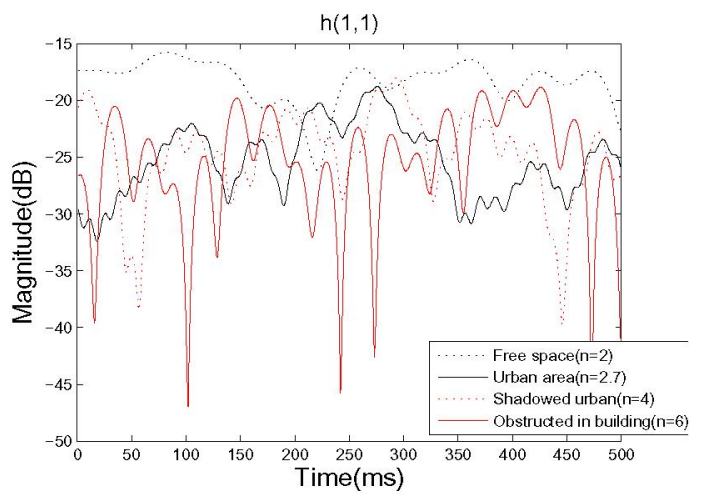

Fig. 3. Evaluation of the impulse response in different environments

where $P_{k}$ is the power assigned to the $k^{t h}$ subchannel and $\lambda_{k}$ is $k^{t h}$ eigenvalue. The maximum capacity is given by

$$
C=\log _{2}\left(1+\rho_{k}\right) \quad \text { bps }
$$

Where the power is allocated to each $k^{\text {th }}$ subchannel by using waterfilling (WF) theorem [3],[2] and each subchannel is filled up to a common level $\mathbf{F}$ :

$$
F=\frac{1}{\lambda_{k}}+P_{1}=\cdots=\frac{1}{\lambda_{k}}+P_{k}
$$

with a limitation on the total transmit Tx power where the total transmitted power $P_{T x}$ is equal to

$$
P_{T x}=\sum_{k=0}^{K} P_{k}
$$

In this case, the largest power is allocated to the $k^{t h}$ subchannel with highest gain.

\section{Simulation Results and Discussion}

The proposed model has been simulated under different environments as described in Table I. Most cases in this work have been examined under urban environment with path loss exponent of $n=4$. Moreover, the distance $D$ has been examined for different values ranging from $500 \mathrm{~m}$ to $2000 \mathrm{~m}$.

Fig. 3 shows the impulse response $h(1,1)$ of the MIMO channel under different environments with different path loss exponents $n$ as shown Table I (column 2). Fig.4 shows the system's behavior in three dimensions: space, time and signal level in $\mathrm{dB}$. The distance is fixed to $D=1500 \mathrm{~m}$, the spreading parameter $a$ is equal to 0.0019 under urban environment.

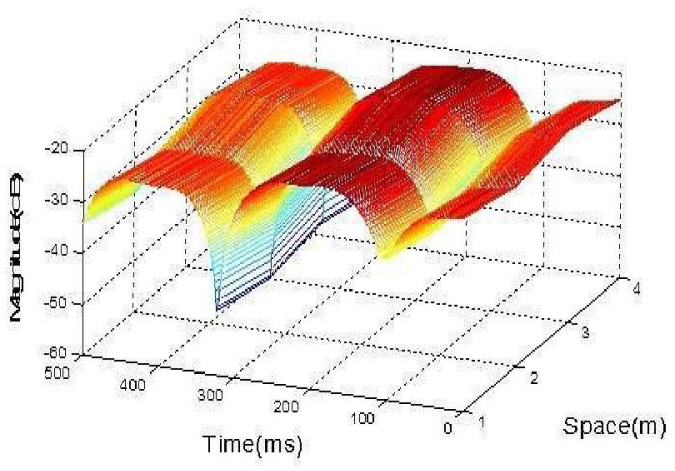

Fig. 4. Space Time Fading of the channel

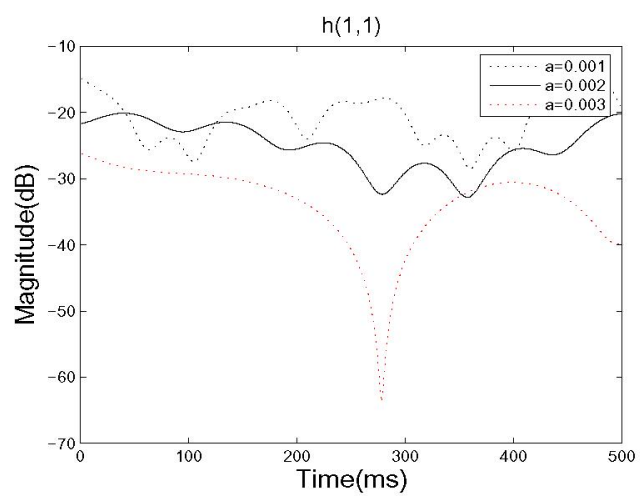

Fig. 5. Impulse response of the channel for different values of spreading factor $a$.

The scattering spread parameter $a$ is chosen to be 0.0019 for most cases. Fig. 5 shows the channel's behavior under different values of $a$.It is also shows the impact of varying the spread parameter $a$ on the signal level in $\mathrm{dB}$, where increasing the spread parameter reduces the angle spread of the scatterers (i.e., reduces the diameter of the scatterers around the mobile). All the parameters mentioned are refereed to the physical environment. In this simulation, the system is employed two antennas at both ends and extended to four in one case to show that capacity is increased with increasing number of antennas.

Fig.6 shows a 3-D graph representing the signal level with the different space distance between the antenna arrays at both transmitter and receiver for fixed distance between the mobile and base station and spread parameter $a$.

Finally, capacity is examined in this paper and the impact of correlation. Fig.7 shows the capacity curve with signal to noise ratio (SNR); and Fig. 8 shows the cumulative distribution function (CDF) vs. capacity for a fixed SNR of $10 \mathrm{~dB}$.

\section{CONCLUSION}

In this paper, we have presented a space time MIMO channel based on geometrical model with a hyperbolically distributed scatterers. The main model provides three important parameters that characterize the channel: the power of the 


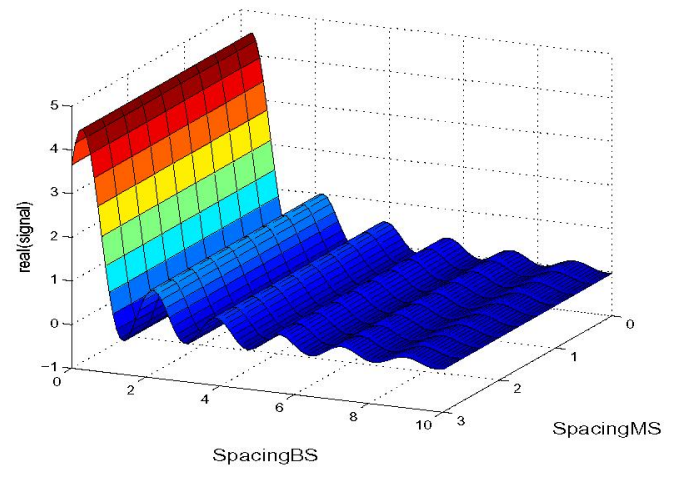

Fig. 6. Envelope of the impulse response of the system vs. $\delta_{T}$ and $\delta_{R}$.

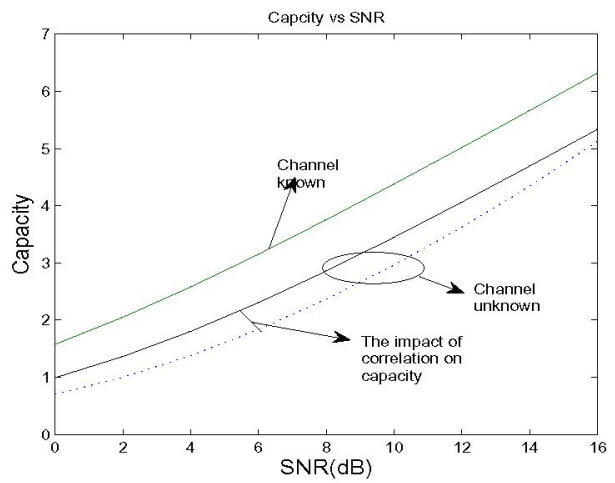

Fig. 7. Capacity fo the channel (in $\mathrm{b} / \mathrm{s} / \mathrm{Hz}$ ) as a function of $\mathrm{SNR}$.

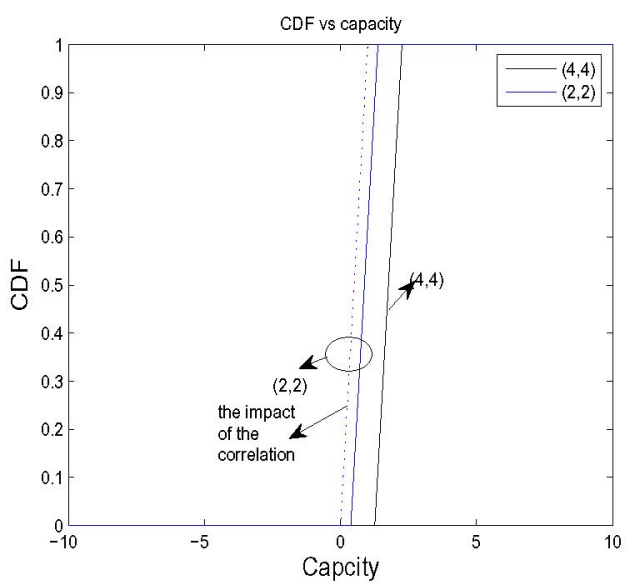

Fig. 8. Cumulative density function (CDF) of the channel as a function of capacity for SNR $=10 \mathrm{~dB}$ ). multipath components, the time of arrival, and the direction of arrival. A uniform linear array antenna is considered at both ends. We have studied the influence of the physical parameters as well as the system along with their impact on the channel capacity. This model has taken Doppler effect and spatial correlation into account.

\section{REFERENCES}

[1] G. Fochini, M. Gans, "On limits of wireless communications in a fading environment when using multiple antennas." Wireless Personal Communications, vol.6, no.3, pp.311-335, 1998.

[2] E. Telatar, "Capcity of mult-antenna Gaussian channels," European Transactions on Telecommunication Related Technologies, vol.10, pp. $585-595,1999$.

[3] C. N. Chuah,J. M. Kahn, and D. Tse, "Capacity of multi-antenna array systems in indoor wireless environment," Proc. of IEEE Global Commun Conf, Sydney, Australia, November 8-12, 1998

[4] K. Yu and B. Ottersten, "Models for MIMO propagation channels: a review," Wireless Communication and Mobile Computing, vol.2, pp.653666,2002

[5] D. Shiu, G. Fochini, M. Gans, and J. Kahn, "Fading correlation and its effect on the capcity of multielement antenna systems,' IEEE Transactions on Communication, vol. 48-3, pp. 502-513, 2000

[6] S. S. Mahmoud, Z. M. Hussain, P. O'Shea, "Geometrical-Based Channel Model with Hyperbolically Distributed scatteres," Multimedia Cyberscape Journal, vol. 2, pp. 1-10, 2004.

[7] S. S. Mahmoud, Z. M. Hussain, P. O'Shea, "Geometrical model for mobile radio channel with hyperbolically distributed scatterers," The 8-th IEEE International Conference on Communication Syatems, vo.1, Singapore, pp. 17-20, Nov.2002

[8] W. C. Jakes, "Microwave propagation," John Wiley, New York, 1971.

[9] M. Stege, J. Jelitto, M. Brozel, G. Fettweis, "A multiple input multiple output channel model for simulation of Tx-and Rx-diversity wireless systems," In Proceedings of the IEEE Vehicular Technology Conference, vol. 2 , pp. 833-839, 2000 .

[10] G. Fochini, "Layered space time architecture for wireless communication in a fading enviroment when using multi-element antennas," Bell Labs Tech. J., pp. 41-59, 1996

[11] A. J. Paulraj and T. Kailath, "Increasing capacity in wireless broadcast systems using distributed transmission/directional reception," Patent no.5 $345599,1944$.

[12] P. Petrus, J. H. Reed, and T. S. Rappaport, "Geometrically based statistical channel model for macro cellular mobile enviroments," in Proc. IEEE Globecom, pp. 1198-1201, 1996.

[13] R. B. Etrel and J. H. Reed, "Angle and time of arrival statistics for circular and elliptical scattering models," IEEE J. Select. Areas Commun., vol. 17, pp. 1829-1840, Nov. 1999.

[14] H. Suzuki, "A statistical model for indoor multipath propagation," IEEE. Trans. Commun., vol. 25, pp. 673-680, July 1977.

[15] R.B.Etrel and J.H.Reed "Angle and time of arrival statistics for circular and elliptical scattering models," IEEE J.Select.Areas Commun. vol.17, pp. 1829-1840, Nov. 1999

[16] S. M. Alamouti, "simple transmit diversity technique for wireless communications," IEEE.Trans. Commun vol. 16, no. 8, pp.1451-1458, Oct. 1988.

[17] V. Tarokh, N. Seshadri, and A. R. Calderbank, "Space Time Code for high wireless communication: performance criterion and code construction," IEEE.Trans. Commun vol. 44, no. 2, pp. 744-765, March 1988. 